\title{
Determination of Uric Acid in Artificial Saliva with Compact AMP3291 Reader and Au Nanoparticles Modified Electrode
}

\author{
Jessica Piedras ${ }^{1}$, Rocio B. Dominguez ${ }^{2}$ and Juan Manuel Gutiérrez ${ }^{1, *(D)}$ \\ 1 Bioelectronics Section, Department of Electrical Engineering, CINVESTAV-IPN, 07360 Mexico City, Mexico; \\ jessicaj.piedrasc@cinvestav.mx \\ 2 CONACyT-CIMAV S.C., Miguel de Cervantes 120, Complejo Industrial Chihuahua, \\ 31136 Chihuahua, CHIH, Mexico; rb.dominguezcruz@gmail.com \\ * Correspondence: mgutierrez@cinvestav.mx; Tel.: +52-55-5747-3800
}

check for updates

Citation: Piedras, J.; Dominguez, R.B.; Gutiérrez, J.M. Determination of Uric Acid in Artificial Saliva with Compact AMP3291 Reader and Au Nanoparticles Modified Electrode. Chemosensors 2021, 9, 73. https:// doi.org/10.3390/chemosensors9040073

Academic Editor: Núria Serrano

Received: 11 March 2021

Accepted: 3 April 2021

Published: 7 April 2021

Publisher's Note: MDPI stays neutral with regard to jurisdictional claims in published maps and institutional affiliations.

Copyright: (c) 2021 by the authors. Licensee MDPI, Basel, Switzerland. This article is an open access article distributed under the terms and conditions of the Creative Commons Attribution (CC BY) license (https:// creativecommons.org/licenses/by/ $4.0 /)$.

\begin{abstract}
Uric acid (UA) is a residual product of purines in the body and has been proposed as a valuable biomarker for Diabetes Mellitus, renal disorder, hypertension and preeclampsia. This work presents a sensing platform for nonenzymatic UA detection using a screen-printed electrode modified with gold nanoparticles (SPE-AuNps) operated with the compact and low-cost amperometric reader AMP3291. This laboratory-made instrument was designed using the analog front end LMP91000 and the microcontroller ESP32; the operational parameters like working potential, acquisition time and dynamic measuring range were configured for UA detection. The whole sensing system (AMP3291+ SPE-AuNps) was evaluated for nonenzymatic sensing of UA, showing a fast response time of $3.5 \mathrm{~s}$, a sensitivity of $0.022 \mu \mathrm{A} \cdot \mu \mathrm{M}^{-1}$, a linear range from 20 to $200 \mu \mathrm{M}\left(\mathrm{R}^{2}=0.993\right)$ and a limit of detection of $11.91 \mu \mathrm{M}$. Throughout, a piece of commercial equipment was used for validation and noticeably the measurements with the AMP3291-based platform showed improved performance, indicating the feasibility of the developed instrument for UA monitoring and potentially for in situ decentralized applications. Finally, artificial saliva was used as model medium exhibiting interesting analytical parameters, encouraging to consider the reported system as a potentially valuable tool for monitoring UA for clinical applications in resource-limited settings.
\end{abstract}

Keywords: uric acid; custom equipment; nonenzymatic; saliva; screen printed electrode

\section{Introduction}

Uric acid (UA) is the end product of purines in the body and a valuable biomarker correlated with gout, Diabetes Mellitus, renal disorder, metabolic syndrome, cardiac conditions, hypertension and preeclampsia [1-3]. Usually, UA can be detected using conventional instrumental techniques in serum $(240-520 \mu \mathrm{M})$ or urine $(1.4-4.4 \mathrm{mM})$ but can also be found at lower concentrations in sweat and saliva [4-6]. The importance of UA as a biomarker, the feasibility of its clinical detection range and the availability of samples for analysis, have encouraged the development of electrochemical sensors (ECS) with advantages like the ease of use, rapid detection, minimal sample requirements and high sensitivity for sensing UA in noninvasive biological fluids like saliva [7]. Although the novel generations of ECS include innovative components such as metallic nanoparticles, conductive polymers and carbon-based materials, the main focus remains on the highly sensitive detection towards the target analyte, with the measurements relying on benchtop potentiostats of high-performance characteristics. However, in many scenarios, the customization and miniaturization of the electrochemical equipment are needed. For example, technologies such as point-of-care (POC) diagnosis or wearable sensors demand simple detection devices and straightforward electronic systems to function outside of a controlled environment [7-9]. Additionally, the World Health Organization has established guidelines for diagnostic tests in limited-resource settings; the criteria coined as ASSURED (affordable, sensitive, specific, user-friendly, rapid and robust, equipment-free or minimal 
equipment, and deliverable to end-users) equally applied to POC systems. [10,11]. Considering that custom sensing platforms allow setting the specific parameters for a defined analyte (e.g., time, current, applied potential), a variety of electronic systems with ECS have been developed for decentralized measurements of glucose [12], $\mathrm{H}_{2} \mathrm{O}_{2}$ [13], dopamine [14], progesterone [15], cortisol [16], zinc [17] and diagnosis of celiac disease [18].

The design of high precision and customized electrochemical equipment have experienced significant progress, especially by taking advantage of the development and decreasing cost of microelectronic fabrication. For potentiostat devices, the first design stage involves controlling the potential applied to the electrochemical cell and registering the generated current in the working electrode. These operations have been performed with different electronic configurations using discrete operational amplifiers like OPA4192 [19], TLV4171 [20], MCP6072 [21] and AD8608 [22]; however, similar results can be obtained with CMOS technology [23] and analog front end (AFE) single chips that simplified circuit complexity like the AD5940 and the LMP91000 [12,13,24]. In the second design stage, the electronic input is linked to microcontroller (MCU) platforms like Arduino, ESP32 [14] or Raspberry Pi [19] to perform digitalization of the acquired signals, synchronize functions and configure internal operational parameters. The MCU also controls the mechanisms to store and present the acquired data by using USB communication protocols to a computer [24], wireless connection [14,18] or even cloud database [17]. Following this general approach, custom equipment has been developed in a simple and compact format to perform electrochemical techniques such as cyclic voltammetry $(\mathrm{CV})[16,25]$, differential pulse voltammetry (DPV) $[13,24]$ and amperometry (AMP) $[7,12]$.

For UA determination, it is common to use either the enzymatic or nonenzymatic detection principle. In the first case, the uricase enzyme (Uox) can be immobilized over a modified electrode to produce allantoin, $\mathrm{H}_{2} \mathrm{O}_{2}$ and $\mathrm{CO}_{2}$ and perform detection based on the reaction of the subproducts over the electrode surface [4]. The main advantage of enzymatic detection is the inherent selectivity of uricase, but the associated drawbacks of complex immobilization procedures, enzyme leaking, poor stability and strong interference of $\mathrm{pH}$ and temperature have to be considered. On the other hand, nonenzymatic detection takes advantage of the oxidation of small organic molecules like UA over conventional electrodes [26]. Then, by incorporating catalytic nanomaterials over the surface, such as multiwalled carbon nanotubes (MWCNT) [27], polymers [28], graphene, graphene oxide (GO) or metallic nanoparticles $[29,30]$, the oxidation potential can be switched toward lower values. In that regard, gold nanoparticles (AuNps) are well known for their catalytic activity, enhancing charge transfer properties, promoting higher conductivity and allowing nonenzymatic detection of target analytes [30-32].

In this work, we present the development of a sensing platform for nonenzymatic detection of UA based on a screen-printed electrode modified with AuNps (SPE-AuNps) and a low-cost and miniaturized amperometric reader based on the AFE LMP91000 and the MCU ESP32. The operation of the designed instrument called AMP3291 was compared with a commercial potentiostat, showing similar performance in critical parameters like detection time, sensitivity and detection limit (LOD). The system also allowed the detection of UA in artificial saliva at working ranges significant for clinical applications.

\section{Materials and Methods}

\subsection{Electronic Components}

All electronic components were acquired from Mouser Electronics. The developed AM3291 is based on the programmable AFE LMP91000 (Texas Instruments, Dallas, TX, USA), the precision low-power 16-bit resolution analog to digital converter (ADC) ADS1115 (Texas Instruments, Dallas, TX, USA) and the MCU ESP32 (Espressif Systems, Shanghai, China). The system was powered using an external $9 \mathrm{~V}$ switching power supply (CP SNT $70 \mathrm{~W} 24 \mathrm{~V} 3 \mathrm{~A}$, Weidmüler, Detmold, Germany) fixed at $5 \mathrm{~V}$ with the linear regulator LM7805CT (Texas Instruments, Dallas, TX, USA). Other passive components and connec- 
tors required to complete the electronic design can be identified in the schematic diagram of Supplementary Figure S1.

\subsection{Reagents and Chemicals}

All reagents were of analytical grade and purchased from Sigma-Aldrich, including UA (CAS 69-93-2), $\mathrm{NaCl}$ (CAS 7647-14-5), $\mathrm{KCl}$ (CAS 7447-40-7), $\mathrm{Na}_{2} \mathrm{HPO}_{4}$ (CAS 7558-79-4), $\mathrm{KH}_{2} \mathrm{PO}_{4}$ (CAS 7778-77-0), $\mathrm{H}_{2} \mathrm{SO}_{4}$ (CAS 7664-93-9) and $\mathrm{HAuCl}_{4}$ (CAS 16961-25-4). Double distilled water was used throughout. Phosphate-buffered saline (PBS) was prepared at 0.1 $\mathrm{M}$ and $\mathrm{pH}$ 7.4. The artificial saliva was obtained from Viarden ${ }^{\mathrm{TM}}$ Laboratories and the composition reported per liter of solution was $\mathrm{NaCl} 0.42 \mathrm{~g}, \mathrm{CaCl}_{2} 0.296 \mathrm{~g}, \mathrm{MgCl}_{2} 0.05 \mathrm{~g}$, $\mathrm{KCl} 1.2 \mathrm{~g}$, NaF $0.42 \mathrm{~g}, \mathrm{C}_{6} \mathrm{H}_{14} \mathrm{O}_{6} 30 \mathrm{~g}, \mathrm{C}_{5} \mathrm{H}_{12} \mathrm{O}_{5} 10 \mathrm{~g}$ and $\mathrm{KH}_{2} \mathrm{PO}_{4} 0.34 \mathrm{~g}$ [33].

The UA solutions were prepared in PBS and artificial saliva at different concentrations departing from a stock solution at $10^{-3} \mathrm{M}$.

\subsection{Apparatus and Equipment}

The electrodeposition of AuNps and the electrochemical characterization of the electrodes were performed using the potentiostat 910 PSTAT mini (Metrohm, The Netherlands). The surface of the electrode was analyzed using scanning electron microscopy (SEM) with a JSM5800LV microscope (JEOL, Japan).

\subsection{Preparation of Electrodes}

A screen-printed electrode (SPE) model 6.1208.110 from Metrohm, consisting of a carbon working electrode of $3 \mathrm{~mm}$ diameter, an $\mathrm{Ag}$ pseudo reference electrode and a carbon counter electrode was used as base. For activation, the bare SPE was pretreated using fixed potentials of +1 and $-1 \mathrm{~V}$ during $150 \mathrm{~s}$ each time. This activation aims to eliminate the presence of the nonconductive materials included during the manufacturing of the SPE that can lead to slow kinetics for heterogeneous electrochemical reactions [34-36]. Then, the SPE was subjected to CV during 20 cycles with potentials from 0 to $+0.8 \mathrm{~V}$ at a scan rate of $100 \mathrm{mV} \cdot \mathrm{s}^{-1}$ using PBS as the electrolyte [34]. The activated SPE was washed with PBS and deionized water prior to gold electrodeposition. For this, the electrode was immersed in a solution of $1 \mathrm{mM} \mathrm{HAuCl}_{4}$ (in $0.5 \mathrm{M} \mathrm{H}_{2} \mathrm{SO}_{4}$ ) during two $\mathrm{CV}$ cycles from -0.5 to $+1 \mathrm{~V}$ at $20 \mathrm{mV} \cdot \mathrm{s}^{-1}$. The resulting SPE modified with gold nanoparticles (SPE-AuNps) was applied for UA determination in the buffer and artificial saliva. A diagram of the described process is shown in Figure 1.

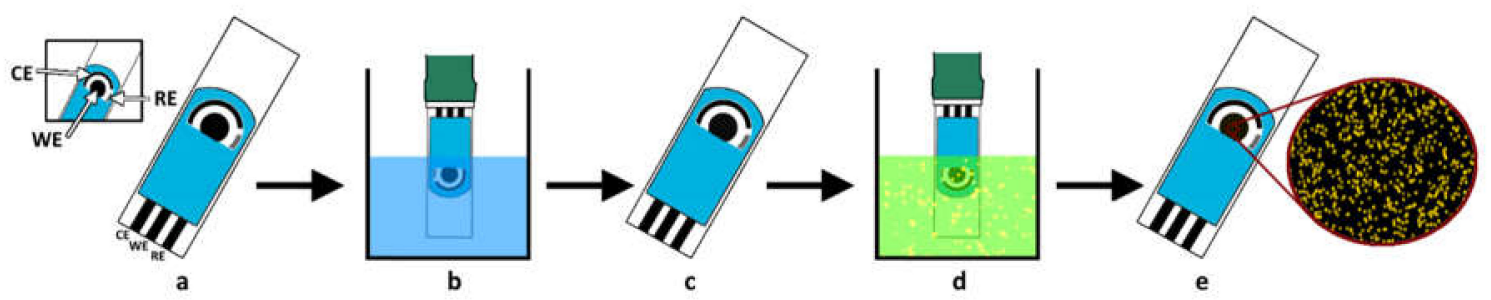

Figure 1. Preparation of screen-printed electrode modified with gold nanoparticles (SPE-AuNps): (a) bare SPE (CE = counter electrode, $\mathrm{WE}=$ working electrode and $\mathrm{RE}=$ reference electrode tequilas), (b) electrochemical pretreatment, (c) activated $\mathrm{SPE},(\mathbf{d})$ electrodeposition of $1 \mathrm{mM} \mathrm{HAuCl}_{4}$ and (e) SPE-AuNps.

\subsection{Design of AMP3291 Instrument}

The core of instrumentation was based on the AFE LMP91000, which allowed the injection of a defined potential into the cell and delivered a voltage proportional to the current measured at the working electrode (WE). For this, the device uses an operational amplifier connected to the counter electrode and a transimpedance amplifier (TIA) that converts the current from the WE to voltage. Then, the ADS1115 was used for digitizing the analog output obtained from the LMP91000. The ADC has four simple channels or 
two differential channels of 16 bits each and an internal reference of $\pm 4.096 \mathrm{~V}$. To handle the voltage range delivered by the LMP91000 with the ADS1115, a programmable gain amplifier (PGA) was integrated to divide the internal reference voltage, achieving six different factor scales: $2 / 3,1,2,4,8$ and 16 . These different voltage ranges operated by the ADS1115 facilitate the software implementation since it is possible to know which voltage resolution can be measured at each scale. The reference voltage (VREF) of $+5 \mathrm{~V}$ resulted in a voltage resolution of $100 \mathrm{mV}$ and a programmed internal zero of $20 \%$ of VREF (equivalent to $+1 \mathrm{~V}$ ) in the LMP91000. The ADS1115 was used in a differential mode, connecting the analog voltage output of the LMP91000 to channel A0 and a fixed voltage of $+1 \mathrm{~V}$ to A1. In this way, the internal zero of the LMP91000 was subtracted, and the ADS1115 registers only the voltage proportional to the current generated in the WE. The hardware configuration and the data acquisition were controlled with the MCU ESP32. The programming of the MCU, LMP91000 and ADS1115 was done through $\mathrm{I}^{2} \mathrm{C}$ protocol using specialized libraries of the Arduino platform available in GitHub repositories, including some adaptations made by the authors to fit the designed hardware (see Supplementary Figure S2a,b). Finally, the data were presented in a graphical user interface (GUI) designed in LabVIEW $2019^{\circledR}$ software. A schematic diagram of the complete system is shown in Figure 2, which simplifies the electronic design of Supplementary Figure S1.

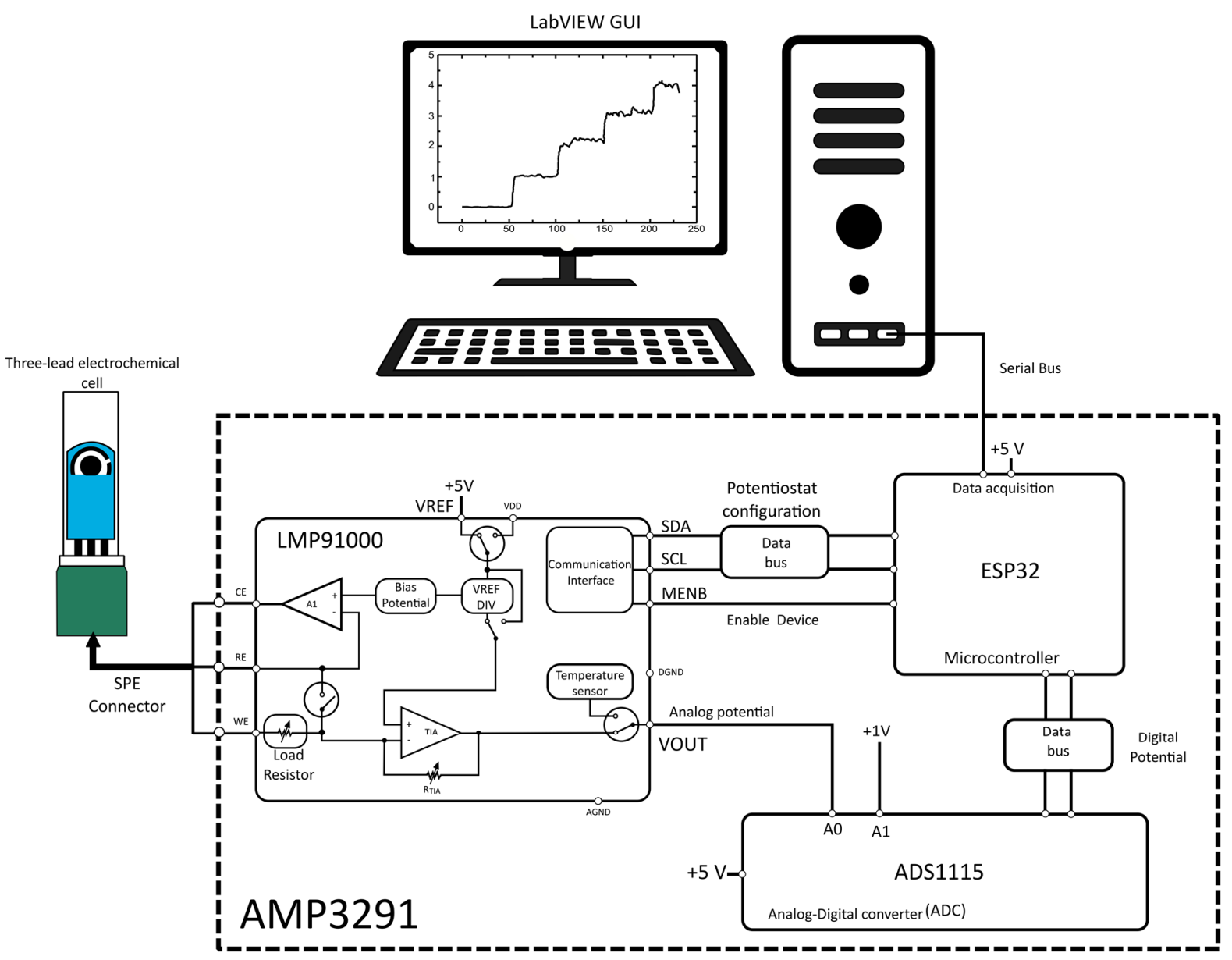

Figure 2. Schematic representation of the custom AMP3291 equipment.

\subsection{Electrochemical Measurements}

The electrochemical performance of the electrodes prior and after AuNps electrodeposition was evaluated with the redox probe $\mathrm{K}_{4}\left[\mathrm{Fe}(\mathrm{CN})_{6}\right]$ at $5 \mathrm{mM}$ in $0.1 \mathrm{M} \mathrm{KCl}$ using CV; these measurements were carried out with the 910 PSTAT mini equipment and potentials from -0.3 to $+0.6 \mathrm{~V}$ at $50 \mathrm{mV} \cdot \mathrm{s}^{-1}$. The amperometric detection of UA was carried out with 
the AMP3291 and the 910 PSTAT mini using a working potential of $+0.3 \mathrm{~V}$ in a range from 20 to $200 \mu \mathrm{M}$ of UA with either PBS or Viarden ${ }^{\mathrm{TM}}$ artificial saliva as solvent.

\section{Results}

\subsection{SPE-AuNps Sensor Characterization}

SEM evaluated the effect of activation and electrodeposition of AuNps over the morphology of the SPE. The view at $\times 750$ in Figure 3a showed the roughness of the surface and the fractures introduced during the electrochemical pretreatment process. The amplified view at $\times 20,000$ in Figure $3 b$ presented a densely covered surface of mainly spherical nanostructures after the electrochemical deposition of AuNps. The particle size histogram in Figure $3 \mathrm{c}$ revealed that $\mathrm{Au}$ nanostructures exhibited mostly diameters of around $56 \mathrm{~nm}$.

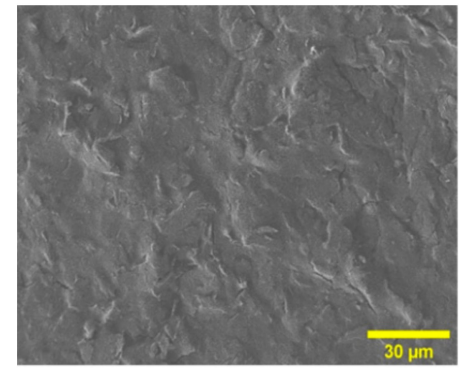

(a)

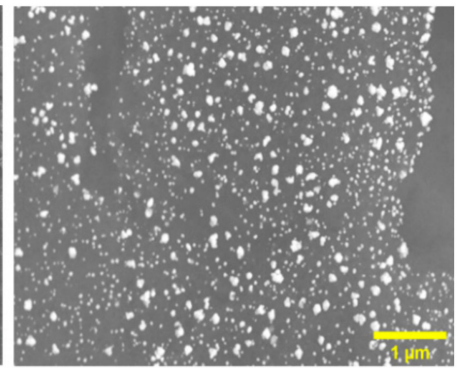

(b)

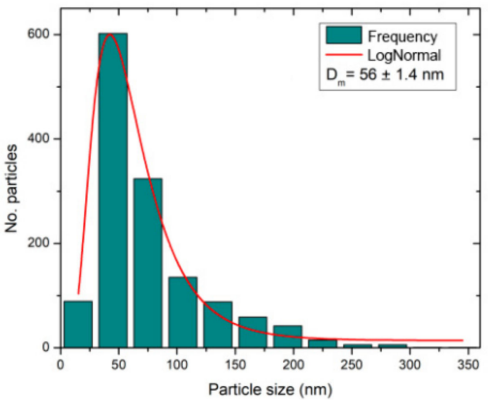

(c)

Figure 3. (a) Activated surface after electrodeposition, (b) a closer view of the resulting AuNps and (c) histogram of AuNps distribution.

The electrochemical performance of the bare SPE and the SPE-AuNps sensor was characterized with $\mathrm{K}_{4}\left[\mathrm{Fe}(\mathrm{CN})_{6}\right]$ redox probe, showing the typical symmetrical peaks of anodic and cathodic currents (Figure 4a). The voltammogram of the bare SPE showed a difference between the anodic and cathodic potentials of $\Delta \mathrm{Ep}=164 \mathrm{mV}$. Simultaneously, the SPE-AuNps decreased the difference to $139 \mathrm{mV}$, corroborating the catalytic activity of the nanostructures and their enhanced charge transfer properties [32]. Then, the ability of both electrodes for UA detection was evaluated using CV at $200 \mu \mathrm{M}$ of UA. In Figure $4 \mathrm{~b}$, the CV for both SPE and SPE-AuNps presented a negligible response in PBS, but after UA addition the SPE showed a defined oxidation peak at $+0.35 \mathrm{~V}$ that shifted to $+0.26 \mathrm{~V}$ in the electrodeposited SPE-AuNps. An increment in current was also registered with $1.853 \mu \mathrm{A}$ for SPE and $2.08 \mu \mathrm{A}$ for SPE-AuNps. A similar behavior was observed when UA was monitored in artificial saliva (Figure 4c), but the oxidation potentials were located at $+0.48 \mathrm{~V}$ for SPE and +0.38 for SPE-AuNps sensor; the current exhibited values of $1.95 \mu \mathrm{A}$ for SPE and $2.09 \mu \mathrm{A}$ for SPE-AuNps. In both cases, the electrodes showed a distinctive current increment after UA oxidation, but the superior performance of AuNps for nonenzymatic sensing allowed detection at lower potentials [31]. 


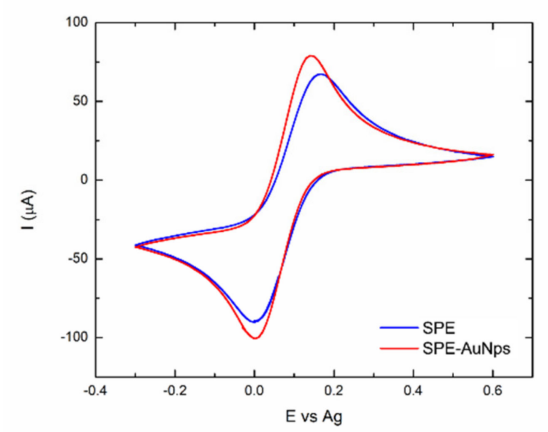

(a)

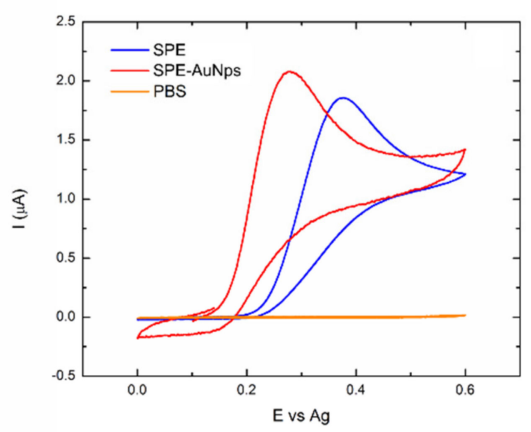

(b)

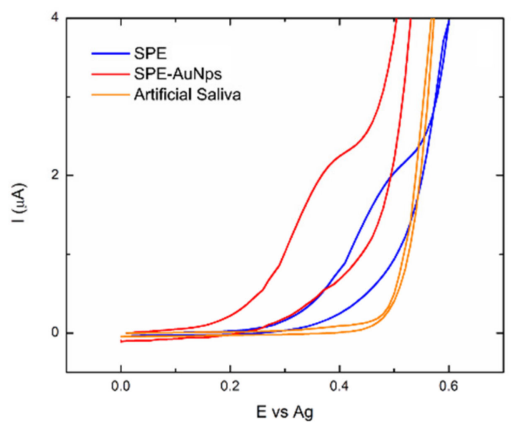

(c)

Figure 4. (a) Cyclic voltammetry (CV) of $1 \mathrm{mM}$ of $\mathrm{K}_{4}\left[\mathrm{Fe}(\mathrm{CN})_{6}\right]$ in bare SPE and SPE-AuNps, (b) detection of both electrodes after UA addition in PBS, (c) detection of both electrodes after UA addition in artificial saliva. Scan rate in all cases was $50 \mathrm{mV} \cdot \mathrm{s}^{-1}$.

\subsection{Evaluation of AMP3291 for UA Detection in PBS}

The AMP3291 system was intended for amperometric measurements of UA in order to reduce complexity of analysis for the final user because they do not need to manipulate the system configuration to make the UA determinations. In this sense, our proposal is a ready-to-use system that does not require any calibration. For example, DPV is commonly applied as a detection technique but adds extra operational parameters to configure it. To avoid this, the internal operation of LMP91000 and ADS1115 has to be programmed appropriately for the optimal performance of the AMP3291 system. Therefore, it was necessary to establish essential parameters related to the amperometric technique, such as the fixed working potential applied to the electrochemical cell, the duration of such applied potential and the frequency between measurements. In this manner, the adequation of the experimental conditions for the determination of UA was carried out by fixed-constant potential determined from the UA oxidation value registered in CV. Given that, VREF in the designed instrumentation is $+5 \mathrm{~V}$; it is possible to corroborate that the necessary source percentage to achieve $\mathrm{a}+0.3 \mathrm{~V}$ fixed-potential was equal to $6 \%$ of VREF (accordingly with the mentioned positions of a voltage divider) as shown below in Equation (1):

$$
V R E F \%=\frac{V_{\text {fixed }}-\text { potential } * 100}{V R E F}
$$

The internal resistor of TIA was selected as $R=7 \mathrm{~K} \Omega$, a value that provided enough gain to obtain a significant output current intensity with minimal presence of noise at the concentration range of interest for most UA clinical determinations. Finally, the measurements were taken every $0.5 \mathrm{~s}$, which was a sufficient sample frequency for the reconstruction of amperometric signals. The reader can consult the Supplementary Material for details about the programmed algorithms in the ESP32. Figure 5 shows the setup for the developed AMP3291.

Once the AMP3291 was configured with a fixed value of $+0.3 \mathrm{~V}$, the whole system was applied for the detection of UA in PBS samples. Figure 6a shows the amperometric I vs. $t$ data obtained after several injections of UA in both pieces of equipment using a bare SPE. The average current recorded for the AMP3291 was $0.3176 \mu \mathrm{A}$ after each injection, while the commercial PSTAT mini registered $0.2783 \mu \mathrm{A}$. This value was considerably increased after electrodeposition, with SPE-AuNps exhibiting $0.4305 \mu \mathrm{A}$ for AMP3291 and $0.3908 \mu \mathrm{A}$ for PSTAT mini (Figure 6b). The enhanced sensitivity of AuNps over UA detection was also evidenced in the differences for the recorded amperometric data in Figure 6c. Noticeably, in all cases, the AMP3291 reader registered a higher current for the same concentration of UA, which can be attributed to the design process that allowed for selection of optimal gain values, therefore achieving comparative values to that obtained with commercial equipment. Moreover, the increased signal after UA addition resulted in higher sensitivity 
for the calibration curve and a lower limit of detection (LOD) for the AMP3291 custom equipment using both SPE and SPE-AuNps electrodes. It is worth noting that although the detection of UA was performed at $+0.3 \mathrm{~V}$, the AMP3291 can be configured to different working potentials, as observed in hardware (Supplementary Figure S3), where detection was performed at $+0.5 \mathrm{~V}$. For the conditions evaluated, the resulting analytical figures of merit are shown in Table 1.

From the reported results, it is possible to establish that amperometric detection with the developed AMP3291 is comparable to results obtained with a high-end portable potentiostat. Likewise, its portability and minimal cost make it an affordable system compared to other commercial systems (see Table 2), with vast possibilities of use in decentralized monitoring applications in settings with limited resources.

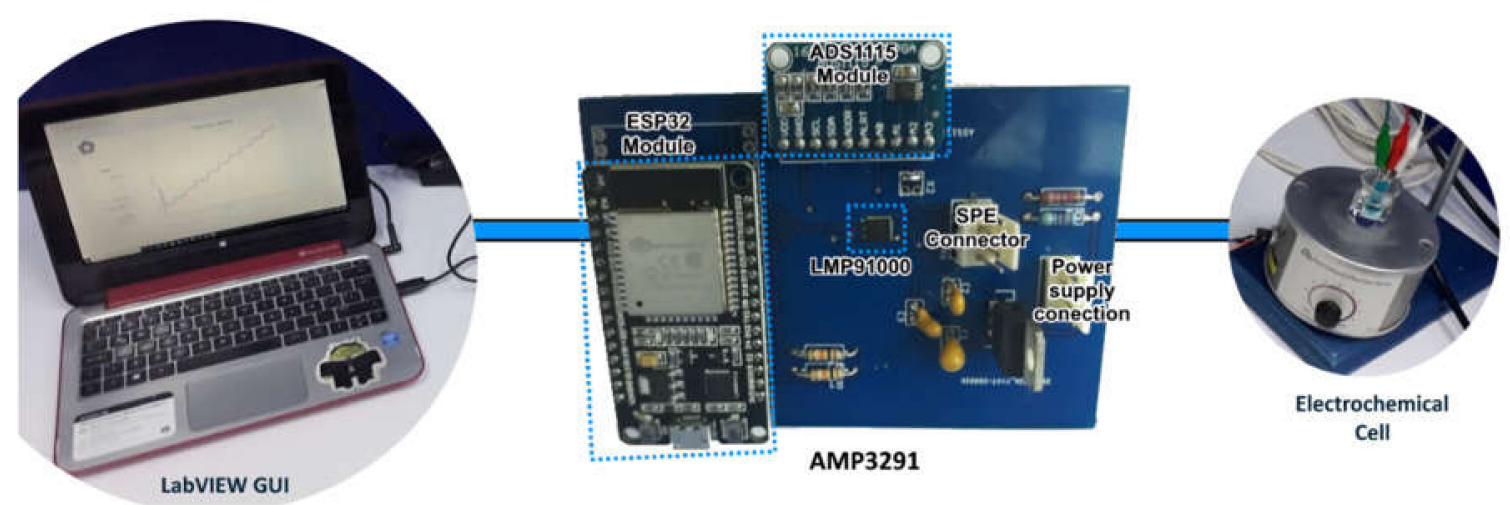

Figure 5. Setup of the sensing platform: AMP3291 reader and the SPE-AuNps.

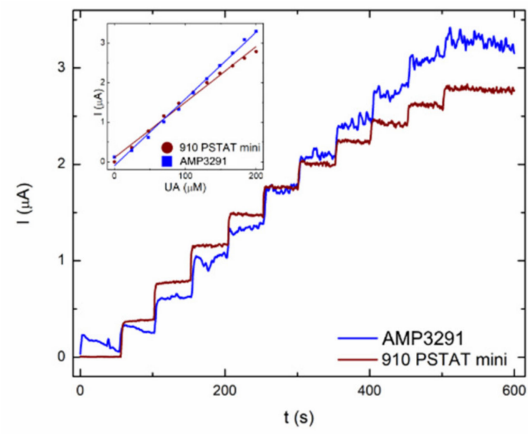

(a)

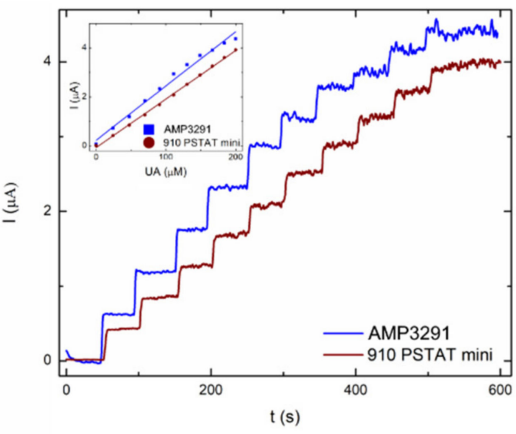

(b)

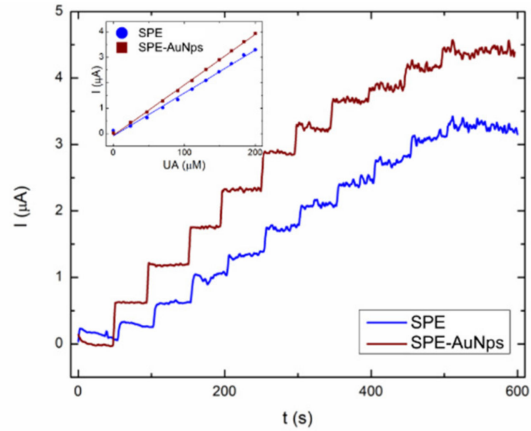

(c)

Figure 6. Comparison of the sensing platform in PBS with AMP3291 and 910 PSTAT mini using (a) SPE, (b)SPE-AuNps and (c) differences in registered amperometric current for SPE and SPE-AuNps in the AMP3291 reader.

Table 1. Analytical figures of merit for AMP3291 and 910 PSTAT mini systems.

\begin{tabular}{ccccccc}
\hline System & $\begin{array}{c}\text { Response Time } \\
{[\mathbf{s}]}\end{array}$ & $\begin{array}{c}\text { Linear Range } \\
{[\mu \mathbf{M}]}\end{array}$ & $\begin{array}{c}\text { Sensitivity } \\
{[\mu \mathbf{A} / \mu \mathbf{M}]}\end{array}$ & $\mathbf{R}^{2}$ & $\begin{array}{c}\text { LOD }^{* *} \\
{[\mu \mathbf{M}]}\end{array}$ & $\begin{array}{c}\text { RSD } \\
{[n=6]}\end{array}$ \\
\hline 910 PSTAT mini-SPE & 3.35 & $20-200$ & 0.014 & 0.999 & 18.63 & $10.53 \%$ \\
\hline 910 PSTAT mini SPE-AuNps & 4.05 & $20-200$ & 0.02 & 0.999 & 12.47 & $18.70 \%$ \\
\hline AMP3291-SPE & 4.1 & $20-200$ & 0.017 & 0.996 & 16.39 & $12.94 \%$ \\
\hline AMP3291-SPE-AuNps & 3.5 & $20-200$ & 0.022 & 0.993 & 11.91 & $1.53 \%$ \\
\hline
\end{tabular}

* Sensitivity defined as the slope of the calibration curve. ${ }^{* *}$ Limit of detection (LOD) defined as $3 \sigma$ of blank/sensitivity. Relative standard deviation (RSD). 
Table 2. Cost comparison between AMP3291 and similar systems.

\begin{tabular}{ccc}
\hline System Model & Unit Price (USD) & Manufactured by \\
\hline 910 PSTAT mini & 4904 & Metrohm, Netherlands \\
\hline C-SENSIT-SM & 930.63 & PalmSens, Netherlands \\
\hline AMP3291 & 76.39 & CINVESTAV, Mexico \\
\hline
\end{tabular}

\subsection{Detection Performance of $U A$ in Artificial Saliva}

Viarden artificial saliva spiked with UA was selected as model medium of biological fluids to evaluate the performance of the sensing platform in real applications. Composition of artificial saliva includes anions and cations like $\mathrm{Na}^{+}, \mathrm{K}^{+}, \mathrm{Mg}^{++}, \mathrm{F}^{-}$and $\mathrm{Cl}^{-}$, and higher viscosity. According to the results observed in $\mathrm{CV}$ and the performance registered in PBS, a working potential of $+0.3 \mathrm{~V}$ in combination with the SPE-AuNps sensor was selected for monitoring. This was mainly due to catalytic activity of the AuNps, which dramatically increased the registered current and enhanced the overall sensing performance compared with bare SPE (Supplementary Figure S4). Figure 7 shows the I vs. t curve obtained with both equipment and the calibration curve related to these data. Once again, the operation of the AMP3291 approach showed enhanced performance in current detection and a lower average of relative standard deviation (RSD) of $6.14 \%$ compared with $14 \%$ of PSTAT mini.

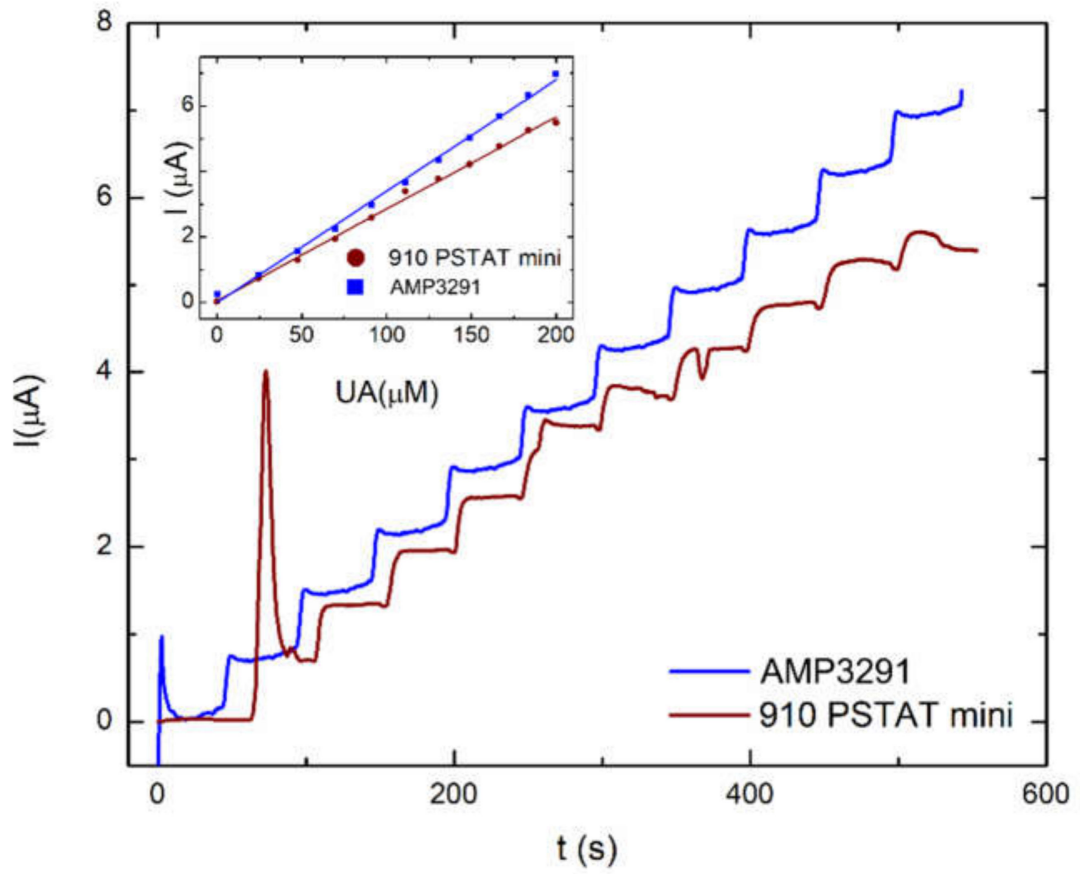

Figure 7. Comparison of the sensing platform in artificial saliva with AMP3291 and 910 PSTAT mini using the SPE-AuNps.

Table 3 presents the analytical parameters of previous works developed for enzymatic and nonenzymatic UA detection using benchtop equipment. Even though some presented exceptional lower LOD [27,37], the high sensitivity was mainly due to the complex surface modification by nanomaterials. Although this represents an advantage, the UA clinical ranges in serum, urine and saliva are significantly higher than those observed in ultrasensitive systems. Thus, to advance these monitoring systems for real applications, the operation in decentralized fashion became as important as improving the analytical parameters. In the AMP3291+SPE-AuNps system presented, the LOD of $14.64 \mu \mathrm{M}$ obtained and the operational working range are in a reasonable agreement with the requirements for UA detection in saliva. Moreover, the simplicity of the designed instrumentation and 
amperometric technique allow the operation outside of a controlled environment. The dependence of an enzymatic system that might be affected by environmental conditions was also avoided by including the nonenzymatic SPE-AuNps sensor.

Table 3. Comparison with similar systems for detection of UA.

\begin{tabular}{|c|c|c|c|c|c|c|c|}
\hline System & $\begin{array}{c}\text { Linear Range } \\
{[\mu \mathrm{M}]}\end{array}$ & $\begin{array}{l}\text { Sensitivity } \\
{[\mu \mathrm{A} / \mu \mathrm{M}]}\end{array}$ & $\begin{array}{l}\text { LOD } \\
{[\mu \mathrm{M}]}\end{array}$ & Equipment & Technique & Sample & Ref. \\
\hline SPE/PB/PPD/Uox & $0-1000$ & 1.08 & - & Custom & AMP & saliva & [7] \\
\hline GCE/PCN/MWCNT & $0.2-20$ & 0.6638 & 0.139 & CHI660D & DPV & serum & [27] \\
\hline SPCEs/Nafion & $62.5-5000$ & 9.366 & 20.8 & Reference 600+ & DPV & PBS & [28] \\
\hline GCE/AuNps & $2.8-57.5$ & 0.121 & 2.8 & CHI760D & DPV & PBS & [29] \\
\hline GCE/AuNps@MoS 2 & $50-4000$ & 0.1611 & 10 & PGSTAT 32 & DPV & PBS & [30] \\
\hline $\begin{array}{c}\text { Pt/Graphene- } \\
\text { Nafion/PANI/Uox-GO }\end{array}$ & $0.01-500$ & - & 0.01 & PARSTAT 2273 & AMP & saliva & [37] \\
\hline flexible sensor $/ \mathrm{MoS}_{2}$ & $10-400$ & 98.3 & 1.16 & CHI660E & AMP & urine & [38] \\
\hline $\mathrm{PGE} / \mathrm{FeS}_{2}$ & $10-725$ & 370 & 6.7 & CHI660E & DPV & urine & [39] \\
\hline SPE-AuNps & $20-200$ & 0.034 & 14.64 & AMP3291 & AMP & artificial saliva & This work \\
\hline
\end{tabular}

Moreover, the model artificial saliva was intended as a design step prior to real biological samples and showed feasibility of the system as a valuable tool for analytical determination in samples of clinical interest in resource limited settings.

\section{Conclusions}

We present a sensing platform based on an SPE-AuNps nonenzymatic sensor coupled with the custom amperometric reader AMP3291 to detect UA. The results suggest that the design of custom instrumentation can contribute greatly to improving the performance of these analytical systems. Moreover, the AMP3291 developed provided similar performance as its counterpart benchtop system, but in a compact, low-cost and simpler fashion. The analytical parameters obtained with the AMP3291+SPE-AuNps system are in reasonable agreement with the detection range of UA in saliva, which suggests the feasibility of moving forward with the system to in situ applications in resource-limited settings.

Future work includes extending current system connectivity through Wi-Fi or Bluetooth communication with a mobile device (tablet or cell phone). This feature could enable the system as a portable UA detector, making its operation closer to a point-of-care testing diagnostic capable of matching the results obtained with commonly used rapid electrochemical analyzers.

Supplementary Materials: The following are available online at https: / www.mdpi.com/article / 10.3390/chemosensors9040073/s1, Figure S1: Schematic diagram for AMP3291 design, Figure S2a: General flow diagram for AM3291 operation, b: Description of the programmed subroutines for AM3291 operation, Figure S3: Detection of uric acid with the AM3291 at +0.5V, Figure S4: Detection of uric acid with the AM3291 at $+0.3 \mathrm{~V}$ in artificial saliva, comparison between SPE and SPE-AuNps.

Author Contributions: Methodology, investigation, validation and figures, J.P.; conceptualization, investigation, supervision and writing-review and editing, R.B.D. and J.M.G. All authors have read and agreed to the published version of the manuscript.

Funding: Jessica Piedras is grateful with the Mexican National Council of Science and Technology for financial support through the 926212 scholarship.

Institutional Review Board Statement: Not applicable.

Informed Consent Statement: Not applicable.

Data Availability Statement: Not applicable.

Acknowledgments: The authors would like to acknowledge the technical assistance of Karla Campos (CIMAV) in SEM and QFB. Isabel Wens Flores (CINVESTAV-IPN) in experimental assays.

Conflicts of Interest: The authors declare no conflict of interest. 


\section{References}

1. El Ridi, R.; Tallima, H. Physiological functions and pathogenic potential of uric acid: A review. J. Adv. Res. 2017, 8, 487-493. [CrossRef] [PubMed]

2. Wang, Q.; Wen, X.; Kong, J. Recent Progress on Uric Acid Detection: A Review. Crit. Rev. Anal. Chem. 2019, 0, 1-17. [CrossRef] [PubMed]

3. Shakarami, A.; Ghafarzadeh, M.; Yari, F.; Fathi, L. Association between maternal serum uric acid and preeclampsia. Arch. Physiol. Biochem. 2020. [CrossRef] [PubMed]

4. Esra, P.; Kılıc, E. A review of enzymatic uric acid biosensors based on amperometric detection. Talanta 2013, 107, $312-323$. [CrossRef]

5. Riis, J.L.; Bryce, C.I.; Matin, M.J.; Stebbins, J.L.; Kornienko, O.; Van Huisstede, L.; Granger, D.A. The validity, stability, and utility of measuring uric acid in saliva. Biomark. Med. 2018, 12, 583-596. [CrossRef]

6. Heikenfeld, J. Let them see you sweat. IEEE Spectr. 2014, 51, 46-63. [CrossRef]

7. Kim, J.; Imani, S.; de Araujo, W.R.; Warchall, J.; Valdés-Ramírez, G.; Paixão, T.R.L.C.; Mercier, P.P.; Wang, J. Wearable salivary uric acid mouthguard biosensor with integrated wireless electronics. Biosens. Bioelectron. 2015, 74, 1061-1068. [CrossRef]

8. Yang, Y.; Song, Y.; Bo, X.; Min, J.; Pak, O.S.; Zhu, L.; Wang, M.; Tu, J.; Kogan, A.; Zhang, H.; et al. A laser-engraved wearable sensor for sensitive detection of uric acid and tyrosine in sweat. Nat. Biotechnol. 2020, 38, 217-224. [CrossRef] [PubMed]

9. Shrivastava, S.; Trung, T.Q.; Lee, N.E. Recent progress, challenges, and prospects of fully integrated mobile and wearable point-of-care testing systems for self-testing. Chem. Soc. Rev. 2020, 49, 1812-1866. [CrossRef]

10. Kosack, C.S.; Page, A.-L.; Klatser, P.R. A guide to aid the selection of diagnostic tests. Bull World Heal. Organ 2017. [CrossRef] [PubMed]

11. Chin, C.D.; Linder, V.; Sia, S.K. Commercialization of microfluidic point-of-care diagnostic devices. Lab Chip 2012, $12,2118$. [CrossRef]

12. Zhang, Z.; Wu, H.; Gao, Y.; Huang, L.; Pan, H.; Du, M. Miniaturised electrochemical analyser for glucose determination based on Chitosan/GOD/Electroreduced graphene oxide sensor. Int. J. Electrochem. Sci. 2020, 15, 2458-2469. [CrossRef]

13. Saygili, E.; Orakci, B.; Koprulu, M.; Demirhan, A.; Ilhan-Ayisigi, E.; Kilic, Y.; Yesil-Celiktas, O. Quantitative determination of $\mathrm{H} 2 \mathrm{O} 2$ for detection of alanine aminotransferase using thin film electrodes. Anal. Biochem. 2020, 591. [CrossRef]

14. Muralidharan, R.; Chandrashekhar, V.; Butler, D.; Ebrahimi, A. A Smartphone-Interfaced, Flexible Electrochemical Biosensor Based on Graphene Ink for Selective Detection of Dopamine. IEEE Sens. J. 2020, 20, 13204-13211. [CrossRef]

15. Serafín, V.; Martínez-García, G.; Aznar-Poveda, J.; Lopez-Pastor, J.A.; Garcia-Sanchez, A.J.; Garcia-Haro, J.; Campuzano, S.; YáñezSedeño, P.; Pingarrón, J.M. Determination of progesterone in saliva using an electrochemical immunosensor and a COTS-based portable potentiostat. Anal. Chim. Acta 2019, 1049, 65-73. [CrossRef] [PubMed]

16. Cruz, A.F.D.; Norena, N.; Kaushik, A.; Bhansali, S. A low-cost miniaturized potentiostat for point-of-care diagnosis. Biosens. Bioelectron. 2014, 62, 249-254. [CrossRef]

17. Nikolaev, K.G.; Kalmykov, E.V.; Shavronskaya, D.O.; Nikitina, A.A.; Stekolshchikova, A.A.; Kosareva, E.A.; Zenkin, A.A.; Pantiukhin, I.S.; Orlova, O.Y.; Skalny, A.V.; et al. ElectroSens Platform with a Polyelectrolyte-Based Carbon Fiber Sensor for Point-of-Care Analysis of Zn in Blood and Urine. ACS Omega 2020, 5, 18987-18994. [CrossRef]

18. Giannetto, M.; Bianchi, V.; Gentili, S.; Fortunati, S.; De Munari, I.; Careri, M. An integrated IoT-Wi-Fi board for remote data acquisition and sharing from innovative immunosensors. Case of study: Diagnosis of celiac disease. Sensors Actuators B Chem. 2018, 273, 1395-1403. [CrossRef]

19. Irving, P.; Cecil, R.; Yates, M.Z. MYSTAT: A compact potentiostat/galvanostat for general electrochemistry measurements. HardwareX 2021, 9, e00163. [CrossRef]

20. Adams, S.D.; Doeven, E.H.; Quayle, K.; Kouzani, A.Z. MiniStat: Development and Evaluation of a Mini-Potentiostat for Electrochemical Measurements. IEEE Access 2019, 7, 31903-31912. [CrossRef]

21. Shen, X.; Ju, F.; Li, G.; Ma, L. Smartphone-based electrochemical potentiostat detection system using pedot: Pss/chitosan/graphene modified screen-printed electrodes for dopamine detection. Sensors 2020, 20, 2781. [CrossRef]

22. Ji, D.; Liu, L.; Li, S.; Chen, C.; Lu, Y.; Wu, J.; Liu, Q. Smartphone-based cyclic voltammetry system with graphene modified screen printed electrodes for glucose detection. Biosens. Bioelectron. 2017, 98, 449-456. [CrossRef]

23. Li, H.; Liu, X.; Li, L.; Mu, X.; Genov, R.; Mason, A.J. CMOS electrochemical instrumentation for biosensor microsystems: A review. Sensors 2017, 17, 74. [CrossRef] [PubMed]

24. Butterworth, A.; Corrigan, D.K.; Ward, A.C. Electrochemical detection of oxacillin resistance with SimpleStat: a low cost integrated potentiostat and sensor platform. Anal. Methods 2019, 11, 1958-1965. [CrossRef]

25. Glasscott, M.W.; Verber, M.D.; Hall, J.R.; Pendergast, A.D.; McKinney, C.J.; Dick, J.E. SweepStat: A Build-It-Yourself, Two-Electrode Potentiostat for Macroelectrode and Ultramicroelectrode Studies. J. Chem. Educ. 2020, 97, 265-270. [CrossRef]

26. Chen, X.; Wu, G.; Cai, Z.; Oyama, M.; Chen, X. Advances in enzyme-free electrochemical sensors for hydrogen peroxide, glucose, and uric acid. Microchim. Acta 2014, 181, 689-705. [CrossRef]

27. Lv, J.; Li, C.; Feng, S.; Chen, S.-M.; Ding, Y.; Chen, C.; Hao, Q.; Yang, T.-H.; Lei, W. A novel electrochemical sensor for uric acid detection based on PCN/MWCNT. Ionics 2019. [CrossRef]

28. Xu, Z.; Zhang, M.-q.; Zou, H.-q.; Liu, J.-s.; Wang, D.-z.; Wang, J.; Wang, L.-d. ding Non-enzymatic electrochemical detection of uric acid with electrodeposited Nafion film. J. Electroanal. Chem. 2019, 841, 129-134. [CrossRef] 
29. Shi, Y.; Wang, J.; Li, S.; Yan, B.; Xu, H.; Zhang, K.; Du, Y. The Enhanced Photo-Electrochemical Detection of Uric Acid on Au Nanoparticles Modified Glassy Carbon Electrode. Nanoscale Res. Lett. 2017, 12, 455. [CrossRef]

30. Sun, H.; Chao, J.; Zuo, X.; Su, S.; Liu, X.; Yuwen, L.; Fan, C.; Wang, L. Gold nanoparticle-decorated MoS2 nanosheets for simultaneous detection of ascorbic acid, dopamine and uric acid. RSC Adv. 2014, 4, 27625-27629. [CrossRef]

31. Antuña-Jiménez, D.; González-García, M.B.; Hernández-Santos, D.; Fanjul-Bolado, P. Screen-printed electrodes modified with metal nanoparticles for small molecule sensing. Biosensors 2020, 10, 9. [CrossRef]

32. Saldan, I.; Dobrovetska, O.; Sus, L.; Makota, O.; Pereviznyk, O.; Kuntyi, O.; Reshetnyak, O. Electrochemical synthesis and properties of gold nanomaterials. J. Solid State Electrochem. 2018, 22, 637-656. [CrossRef]

33. Machado-López, M.M.; Faure, J.; Espinosa-Medina, M.A.; Espitia-Cabrera, M.I.; Contreras-García, M.E. Enhanced Corrosion Resistance in Artificial Saliva of Ti6Al4V with ZrO 2 Nanostructured Coating. J. Electrochem. Soc. 2015, 162, D3090-D3100. [CrossRef]

34. Huang, D.; Cheng, Y.; Xu, H.; Zhang, H.; Sheng, L.; Xu, H.; Liu, Z.; Wu, H.; Fan, S. The determination of uric acid in human body fluid samples using glassy carbon electrode activated by a simple electrochemical method. J. Solid State Electrochem. 2015, 19, 435-443. [CrossRef]

35. Rana, A.; Baig, N.; Saleh, T.A. Electrochemically pretreated carbon electrodes and their electroanalytical applications-A review. J. Electroanal. Chem. 2019, 833, 313-332. [CrossRef]

36. González-Sánchez, M.I.; Gómez-Monedero, B.; Agrisuelas, J.; Iniesta, J.; Valero, E. Highly activated screen-printed carbon electrodes by electrochemical treatment with hydrogen peroxide. Electrochem. commun. 2018, 91, 36-40. [CrossRef]

37. Liao, C.; Mak, C.; Zhang, M.; Chan, H.L.W.; Yan, F. Flexible Organic Electrochemical Transistors for Highly Selective Enzyme Biosensors and Used for Saliva Testing. Adv. Mater. 2015, 27, 676-681. [CrossRef] [PubMed]

38. Sha, R.; Vishnu, N.; Badhulika, S. MoS2 based ultra-low-cost, flexible, non-enzymatic and non-invasive electrochemical sensor for highly selective detection of Uric acid in human urine samples. Sens. Actuators B Chem. 2019, 279, 53-60. [CrossRef]

39. Sha, R.; Vishnu, N.; Badhulika, S. FeS2 Grown Pencil Graphite as an In-expensive and Non-enzymatic Sensor for Sensitive Detection of Uric Acid in Non-invasive Samples. Electroanalysis 2019, 31, 2397-2403. [CrossRef] 